\title{
CRISPR-Cas Systems Features and the Gene-Reservoir Role of Coagulase-Negative Staphylococci
}

\author{
Ciro C. Rossi*, Thaysa Souza-Silva, Amanda V. Araújo-Alves and \\ Marcia Giambiagi-deMarval* \\ Instituto de Microbiologia Paulo de Góes, Universidade Federal do Rio de Janeiro, Rio de Janeiro, Brazil
}

\section{OPEN ACCESS}

Edited by:

Vasco Ariston De Carvalho Azevedo,

Universidade Federal de Minas

Gerais, Brazil

Reviewed by:

Pamela Del Carmen Mancha-Agresti, Universidade Federal de Minas

Gerais, Brazl

Soo Rin Kim

Kyungpook National University,

South Korea

${ }^{*}$ Correspondence:

Ciro C. Rossi ciro.rossi@hotmail.com

Marcia Giambiagi-deMarval

marciagm@micro.ufr.jbr

Specialty section:

This article was submitted to

Evolutionary and Genomic

Microbiology,

a section of the journal

Frontiers in Microbiology

Received: 18 May 2017

Accepted: 31 July 2017

Published: 15 August 2017

Citation

Rossi CC, Souza-Silva T,

Araújo-Alves AV and

Giambiagi-deMarval M (2017)

CRISPR-Cas Systems Features

and the Gene-Reservoir Role

of Coagulase-Negative Staphylococci.

Front. Microbiol. 8:1545.

doi: 10.3389/fmicb.2017.01545
The claimed role of gene reservoir of coagulase-negative staphylococci (CoNS) could be contradicted by estimates that CRISPR/Cas systems are found in the genomes of $40-50 \%$ of bacteria, as these systems interfere with plasmid uptake in staphylococci. To further correlate this role with presence of CRISPR, we analyzed, by computational methods, 122 genomes from 15 species of CoNS. Only 15\% of them harbored CRISPR/Cas systems, and this proportion was much lower for S. epidermidis and S. haemolyticus, the CoNS most frequently associated with opportunistic infections in humans. These systems are of type II or III, and at least two of them are located within SCCmec, a mobile genetic element of Staphylococcus bacterial species. An analysis of the spacers of these CRISPRs, which come from exogenous origin, allowed us to track the transference of the SCCmec, which was exchanged between different strains, species and hosts. Some of the spacers are derived from plasmids described in Staphylococcus species that are different from those in which the CRISPR are found, evidencing the attempt (and failure) of plasmid transference between them. Based on the polymorphisms of the cas 1 gene in CRISPRs of types II and III, we developed a multiplex polymerase chain reaction (PCR) suitable to screen and type CRISPR systems in CoNS. The PCR was tested in 59 S. haemolyticus strains, of which only two contained a type III cas1. This gene was shown to be expressed in the exponential growth, stationary phase and during biofilm formation. The low abundance of CRISPRs in CoNS is in accordance with their role as gene reservoirs, but when present, their spacers sequence evidence and give an insight on the dynamics of horizontal genetic transfer among staphylococci.

Keywords: CRISPR, cas genes, CoNS, coagulase-negative staphylococci, horizontal gene transfer

\section{INTRODUCTION}

Coagulase-negative staphylococci (CoNS) were for a long time considered as harmless residents of the normal microbiota of skin and mucous membranes of humans, but are increasingly being recognized as central causative agents of healthcare-associated infections (Becker et al., 2014; Asaad et al., 2016). Among them, Staphylococcus epidermidis and Staphylococcus haemolyticus have become two major causes of nosocomial infections, which are difficult to eradicate given their ability to acquire multiresistance against available antimicrobial agents and capacity to form biofilms on indwelling medical devices (Vuong and Otto, 2002; Czekaj et al., 2015). 
The establishment of biofilms also provide an environment of high cell density, increased genetic competence and availability of mobile genetic elements that altogether make an ideal setting for horizontal transference of resistance genes (Flemming et al., 2016). In fact, evidences show that CoNS may act as reservoirs of genes that may be transferred between related and to more pathogenic bacteria, such as Staphylococcus aureus, thus enhancing its potential to resist antimicrobial treatment (Otto, 2013; Rossi et al., 2016a). The evidences for transfer of important elements, such as plasmids and the staphylococcal cassette chromosome mec (SCCmec, which harbors the mecA gene coding for an alternative penicillin binding protein), from CoNS to $S$. aureus are quite strong as observed by sequence homology (Barbier et al., 2010; Otto, 2013).

Conversely, horizontal gene transfer is greatly limited in staphylococci by the presence of clustered regularly interspaced short palindromic repeats - CRISPR (Marraffini and Sontheimer, 2008; Cao et al., 2016), which are believed to be present in approximately $40-50 \%$ and $90 \%$ of bacterial and archaeal genomes, respectively (Sorek et al., 2008).

As suggested by its name, CRISPR is an array of short (24-47 bp) direct repeats (DRs), which are separated from each other by equally short segments of DNA derived from previous exposure to foreign DNA, specially a virus or plasmid (van der Oost et al., 2014). Functional CRISPR systems are always closely accompanied by a cluster of CRISPR-associated proteins (Cas), each in charge of different functions for the system. The complex formed by the Cas1 and Cas2 proteins recognizes fragments of an invader genetic material, previously processed by proteins of the cell repair system. These fragments are incorporated as "spacers" in the bacterial/archaeal genome, within the CRISPR array (Amitai and Sorek, 2016), which is then transcribed as a large transcript containing several spacers separated by palindromic DRs. The transcript is processed into small CRISPR RNAs (crRNA) containing a spacer and a hairpin structure, used for interference in a subsequent invasion of a mobile genetic element containing the same or a sequence similar to the one in the crRNA (Barrangou and Horvath, 2017).

Based on the Cas proteins involved in each step of CRISPR adaptation, expression, maturation and interference, two classes and six types of CRISPR systems are currently recognized. While in class I (comprising types I, III, and IV) systems targeting is performed by multiprotein complexes, in class II (types II, V, and VI) this role is played by a single protein (Barrangou and Horvath, 2017). Interference by type II CRISPR is performed by Cas9 and associated RNAs. Because of its simplicity, this system has been vastly adapted to be used as a tool for mostly modifying sequences and/or controlling expression of target genes (Luo et al., 2016). However, some particularities of Cas9 of different sources, such as the need of recognizing specific sequences - the protoadjacent motif, PAM - and the difficulties in applying this modification system in prokaryotes, are boosting the use of Cas proteins of other types and the search for new systems (Luo et al., 2016; Burstein et al., 2017; Cloney, 2017).

To further understand the importance of coagulase negative staphylococci as gene reservoirs and the relationship between this role and the presence of CRISPR within the bacterial genome, this work aimed to identify and characterize CRISPR systems in several CoNS of species that have been described in humans, as well as establishing an efficient detection method, evaluating its abundance and expression.

\section{MATERIALS AND METHODS}

\section{CoNS Genomes Analyzed}

A total of 122 sequenced genomes available from GenBank (Supplementary Table S1) were used for the computational analysis. They belong to 15 different species of coagulase negative Staphylococcus that have been detected in humans, among other sources (Becker et al., 2014): S. auricularis $(n=1), S$. capitis $(n=7)$, S. epidermidis $(n=34)$, S. haemolyticus $(n=32)$, S. hominis $(n=6)$, S. lugdunensis $(n=9), S$. massiliensis $(n=1)$, S. pettenkoferi $(n=1)$, S. saprophyticus $(n=8)$, S. schleiferi $(n=5)$, S. sciuri $(n=1)$, S. simulans $(n=3)$, S. succinus $(n=1)$, $S$. warneri $(n=10)$, and $S$. xylosus $(n=3)$.

\section{CRISPR Search and Characterization}

All the 122 CoNS genomes were analyzed with the CRISPRFinder tool (Grissa et al., 2007) for the search of possible CRISPR structures. Then, the loci of those genomes that were positive for CRISPR candidates were collected and further annotated with Artemis (Rutherford et al., 2000) for the search of cas genes, and with ISFinder (Siguier et al., 2012) for the search of insertion sequences surrounding the system.

\section{Direct Repeats and Spacer Analysis}

The DRs of every CRISPR found were aligned using Clustal Omega (Sievers and Higgins, 2014); then a phylogenetic inference was done using Mr. Bayes 3.2 (Ronquist et al., 2012). The alignment was also used to build sequence logos with Weblogo 3.0 (Crooks et al., 2004). Secondary structure of each DR was predicted by RNAFold (Gruber et al., 2008). Additionally, the origin of every spacer sequence was investigated by nucleotide BLAST against the NCBI's database.

\section{CRISPR Screening in Clinical Strains}

The sequences of the cas1 gene from all the CRISPR-positive genomes were aligned by Clustal Omega and the best conserved regions were analyzed to design the oligonucleotide pairs cas1IIF (5'-AAT ATA GAG GGC CAA GCG GC-3')/cas1IIR (5'-CGC ATG CAG CAA GTT AAT CAG C-3') to detect a 324 bp fragment of the cas 1 gene from type II CRISPR systems, and cas1IIIF (5' - TGT TAC TGC GAA GGA AAA TAG C-3')/cas1IIIR (5'-CGT CCA CGT TTA AAT TGT TTG CC-3'), to detect a $471 \mathrm{bp}$ cas 1 gene of type III CRISPR systems. Both cas1IIF/R and cas1IIIF/R primer pairs were used in a multiplex polymerase chain reaction (PCR) reaction containing $2 \mathrm{U}$ of the GoTaq G2 DNA Polymerase (Promega, United States) in the enzyme buffer, containing $0.2 \mathrm{mM}$ of each dNTP, $0.2 \mathrm{mM}$ of each primer and $0.1 \mathrm{mM}$ of the primer pair 16S rRNAF/R (Chen et al., 2013) as an endogenous control. Screening was performed in 59 clinical strains of Staphylococcus haemolyticus previously analyzed by our group and shown to be phenotypic and genetically diverse 
(Barros et al., 2012; Rossi et al., 2016b). Amplification was performed in a T100 Thermal Cycler (BioRad, United States) with the following cycle parameters: initial denaturation at $95^{\circ} \mathrm{C}$ for $3 \mathrm{~min}$, followed by 30 cycles $\left(95^{\circ} \mathrm{C}\right.$ for $30 \mathrm{~s}, 50^{\circ} \mathrm{C}$ for $1 \mathrm{~min}$, and $72^{\circ} \mathrm{C}$ for $1 \mathrm{~min}$ ) and $72^{\circ} \mathrm{C}$ for $3 \mathrm{~min}$. The reaction products were analyzed after electrophoresis in 1.0\% agarose gel. Staphylococcus epidermidis RP62A (Gill et al., 2005) was used as a positive control for type III-CRISPR cas 1 and S. epidermidis $10 \mathrm{~L}$ (Rossi et al., 2017); as a positive control for type II-CRISPR cas1.

\section{Real Time Polymerase Chain Reaction}

The expression of cas 1 was evaluated in triplicate by RT-PCR in Staphylococcus haemolyticus clinical strains positive for the gene. Overnight cultures grown on Brain Heart Infusion (BHI-BD, France) agar plates were used to prepare bacterial suspensions using a $0.5 \mathrm{McFarland}$ turbidity standard. The bacterial suspensions were then diluted in 1:100 fresh BHI and incubated at $37^{\circ} \mathrm{C}$ under agitation $(150 \mathrm{rpm})$ for $4 \mathrm{~h}$ (exponential growth), $16 \mathrm{~h}$ (stationary phase) and $48 \mathrm{~h}$ for the formation of biofilms in the walls of the glass flasks used for cultivation. Cells were then harvested and ressuspended in RNAlater (Thermo Fisher Scientific, United States) and kept at $-20^{\circ} \mathrm{C}$ until RNA extraction. RNA extraction was performed with the RNAeasy mini kit (Qiagen, Germany) according to the manufacturer's instructions, with cells being previously disrupted with Lysing Matrix Tubes B (MP Biomedicals, United States) in a Mini-beadbeater-24 (BioSpec, United States) in three rounds of $40 \mathrm{~s}$. RNA quantity and quality were evaluated by spectrophotometry and gel electrophoresis, respectively. Then, RNA was treated with RQ1 RNAse-Free DNAse (Promega, United States), following the manufacturer's instructions. Complete removal of DNA was evaluated by PCR with primers for the $r p o B$ gene, rpoB-2643F and rpoB-3241R, with amplification parameters as suggested by the author of the primers (Drancourt and Raoult, 2002). cDNA first strand synthesis was performed with the DNAse-treated RNA, using the ImProm-II ${ }^{\mathrm{TM}}$ Reverse Transcription System (Promega, United States). Then, a PCR reaction was conducted with the primer pair cas1IIIF/cas1IIIR, $1 \mathrm{U}$ of GoTaq G2 DNA Polymerase (Promega, United States) in the enzyme buffer, containing $0.2 \mathrm{mM}$ of each dNTP and $0.2 \mathrm{mM}$ of each primer. Amplification was done as described above. The reaction products were analyzed after electrophoresis in 1.0\% agarose gel. As negative controls, we used RNA that was subjected to the same reactions of cDNA synthesis, but without the addition of the Reverse Transcriptase.

\section{RESULTS}

\section{CRISPR/Cas Systems in CoNS}

A total of 122 public available genomes, belonging to 15 strains of coagulase negative staphylococci species that have already been described in the human microbiota (among other sources), were analyzed for the presence of CRISPR-like sequences by the CRISPRfinder software. Among them, 32 strains were suspicious of containing CRISPR/Cas systems
(Supplementary Table S1). However, many of these sequences were too small (comprising three DRs and two spacers), and most of them are not adjacent to cas genes. Therefore, these repetitive sequences are not included in real CRISPR/Cas systems. Overall, only $18(15 \%)$ strains, belonging to eight species (S. capitis, S. epidermidis, S. massiliensis, S. schleiferi, S. haemolyticus, S. lugdunensis, S. simulans, and S. warneri) contain CRISPR/Cas systems, characterized by 3 (S. lugdunensis M23590) to 37 (S. schleiferi 1360-13) DRs and an adjacent cluster of cas genes (Figure 1 and Supplementary Figure S1). The proportion is even lower in CoNS that act as important human pathogens: among the S. epidermidis strains, only 9\% (3/34) of them presented characteristic CRISPR/Cas systems, and among S. haemolyticus; only $3 \%(1 / 32)$.

These systems are all complete and are either of type II, containing the cas 1, cas 2 , and cas 9 genes; or of type III, which also contains the universal cas $1-2$ genes, in addition to cas6, cas10, and csm2-6. Curiously, some systems (the type III ones from S. capitis CR03 and S. schleiferi TSCC54) contain repetitive sequences both up and downstream the cas-gene cluster, although one of them seems to be more active than the other, given the difference in the number of spacers presented. In addition, two strains contain two independent CRISPR/Cas systems in their genome: while the S. simulans FDAARGOS_124 strain contains two type II systems, the S. schleiferi TSCC54 strain contains one type II system (like all other strains from this species), but harbors an additional system, of type III.

\section{CRISPR/Cas Systems Exchange between Species}

The presence of a CRISPR/Cas system in S. schleiferi TSCC54 different from the one that is common for the species (Figure 1) lead us to investigate whether they could have had different origins. For that purpose, we first analyzed the DRs of each CRISPR found and made a Bayesian inference of the phylogeny of those sequences, which are usually conserved among species. Most DR formed groups in a speciesdependent manner, in addition to defining two clades: one of type II CRISPR system DR, and other from type III (Figure 2A).

All the DR from type III systems have very conserved regions containing the motif CCCC, separated by eight nucleotides from a GGGG stretch. Both sequences are likely to interact with one another, to form the typical hairpin structures that are both important for the initial processing of the CRISPR transcript and the interaction of the CRISPR small RNA (crRNA, involved in the interference process) with the Cas proteins. Although the same motifs are not observed in the DR from type II systems, which are more varied, all the DR are also capable of forming the typical secondary structures (Figure 2B and Supplementary Figure S2).

The DR from S. schleiferi TSCC54 type III system grouped with the DR from $S$. capitis CR03, which raised the hypothesis of a horizontal transference between them. To investigate the location of CRISPR loci within mobile genetic elements, we first searched for insertion sequences in the vicinity of these 


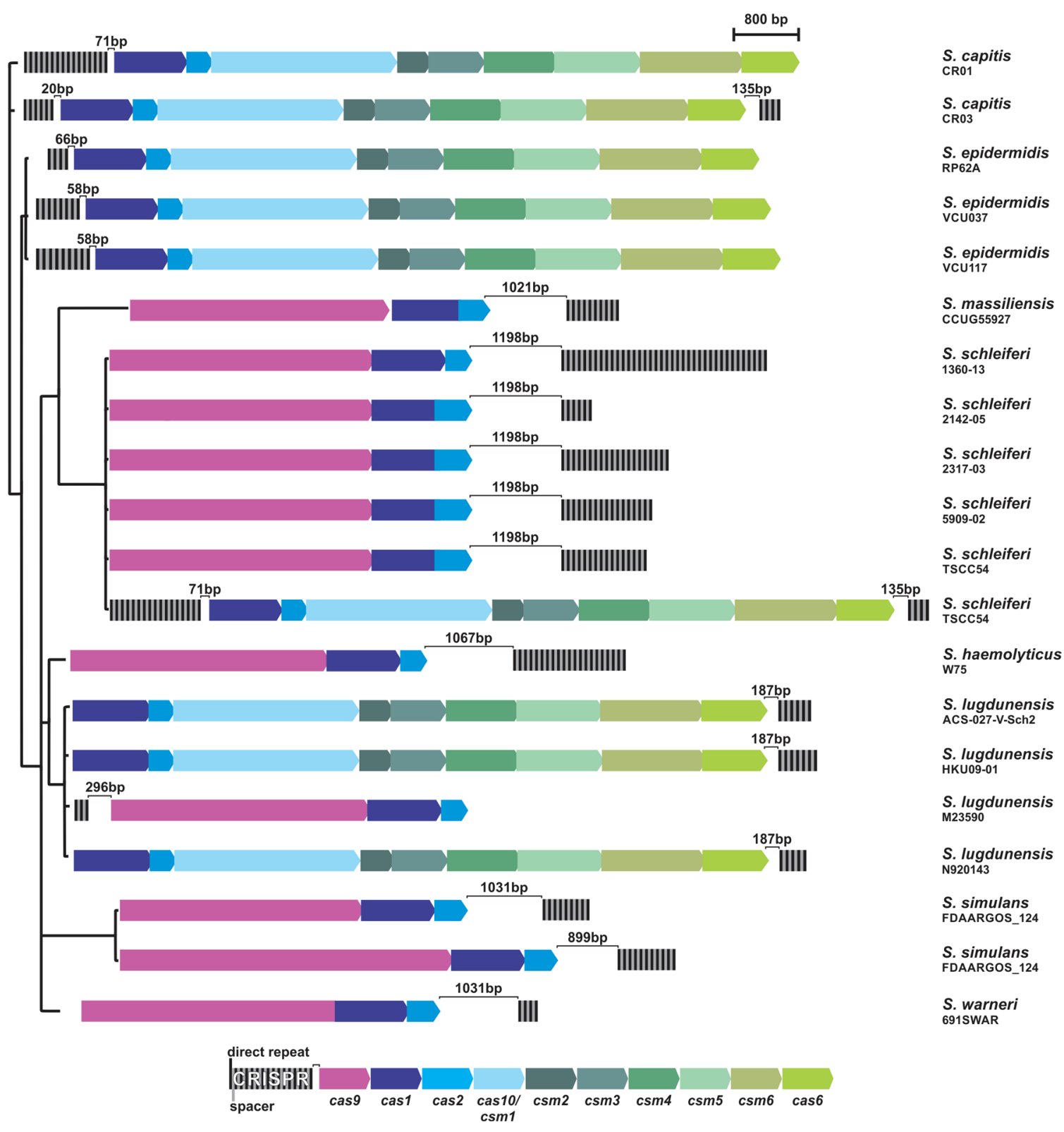

FIGURE 1 | CRISPR/Cas systems in coagulase-negative staphylococci. Black and gray strips represent the direct repeats (DRs) and spacers of each CRISPR (respectively) and arrows represent the genes for each Cas protein. For comparison purposes, strains CRISPRs were organized in a three built from the Bayesian inference of the phylogeny of each strain 16S rRNA sequence.

elements. Many of the systems found in here are nearby insertion sequences (Supplementary Table S2), but an in depth analysis and annotation of genes around the CRISPR/Cas systems of S. schleiferi TSCC54 and S. capitis CR03 showed that they both are located within SCCmec elements (Figure 3A). These elements display an almost identical region (98\% of identity) that is also very similar to a SCCmec previously described for the S. aureus strain 80BA02176 (Golding et al., 2012). Surprisingly, these three systems share most of their spacer sequences, evidencing a common origin (Figure 3B). Given the number and array of spacers, we hypothesize that this
CRISPR/Cas element was originally from a $S$. capitis strain that diverged into two different strains (Figure 3C): (i) one that acquired the spacers S14 and S15, originating the S. capitis CR01 strain and (ii) another that acquired the spacers S16 and S18-S22, originating the strain that served as the donor of the CRISPR-containing SSCmec to S. aureus 80BA02176 (which incorporated the S17 spacer and lost S08-S09) and to S. schleiferi TSCC54 (which acquired S23-24 and lost S03-S04). It is also possible that other intermediate strains were involved in this process of SSCmec and CRISPR/Cas systems exchange process. 
A

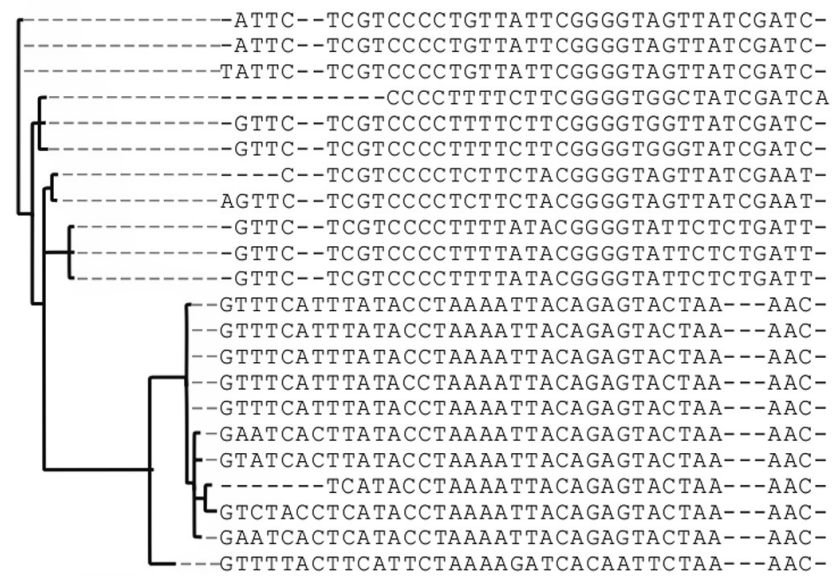

S. capitis CR01

S. schleiferi TSCC 54

S. capitis $\mathrm{CRO} 3$

S. epidermidis VCU037

S. epidermidis VCU117

S. capitis $\mathrm{CRO} 3$

S. schleiferi TSCC54

S. Iuqdunens is ACS-027-V-Sch2

S. Iuqdunensis HKU09-01

S. Iuqdunensis N920143

S. schleiferi TSCC 54

S. schleiferi 5909-02

S. schleiferi 2317-03

S. schleiferi 2142-05

S. schleiferi 1360-13

S. massiliensis CCUG55927

S. Iugdunensis M23590

S. warneri 691SWAR

S. haemolyticus $W 75$

S. simulans FDAARGOS124

S. simulans FDAARGOS 124
S. epidermidis RP62A

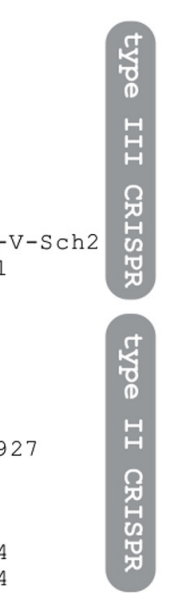

B

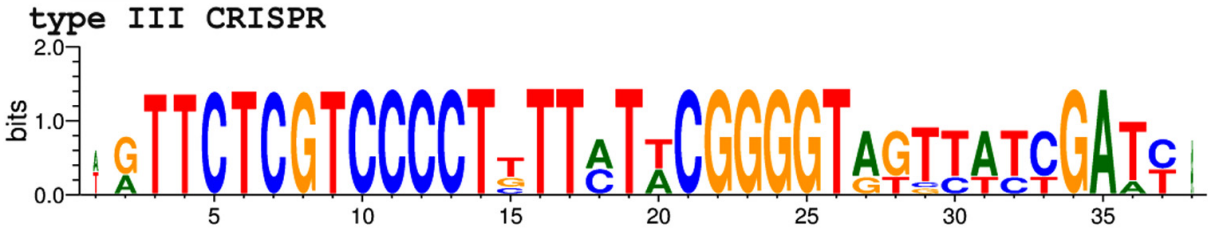

type II CRISPR

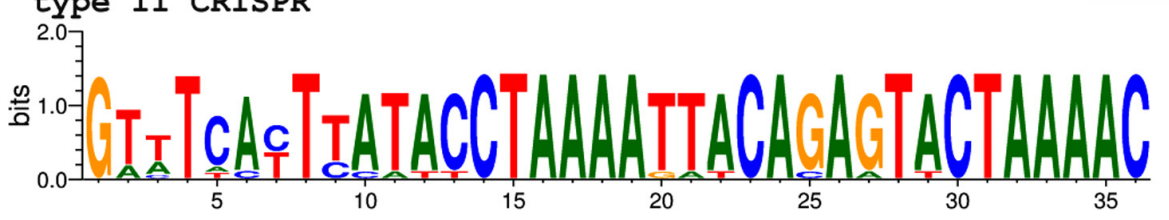

FIGURE 2 | Sequence diversity of the DRs of coagulase-negative staphylococci CRISPRs. (A) Bayesian inference of the phylogeny of each strain DR sequence. Asterisks indicate identical bases in the sequence alignment. (B) Sequence logo of DR of type II and type III CRISPR systems.

\section{The Origin of CoNS Spacers}

Considering that some spacers are identical between the S. capitis CR01 and S. schleiferi TSCC54 strains and that spacers were found repeatedly within the same species (among S. schleiferi strains) and even in the same CRISPR of a single strain (S. massiliensis CCUG55927), there are overall 194 unique spacer sequences. Among them, the great majority (93\%) are from an unknown origin, as they do not present homology with any sequence available from GenBank. Only 13 (7\%) sequences are known, of which seven (4\%) are identical to a variety of plasmids found in different species belonging to the Staphylococcus genus (Supplementary Table S3). A spacer from S. haemolyticus W75 (shae20), for example, has a sequence that is identical to that of the S. aureus plasmid pSAM12-0145 (GenBank ID KU521355.1) and the S. cohnii plasmid pHK01 (GenBank ID KC820816.1). The remaining six (3\%) spacers originally may have come from bacteriophages (Supplementary Table S3), some of which were isolated from other Staphylococcus species.

\section{A Multiplex PCR for Screening and Typing CRISPR Systems in CoNS}

As well as having very distinct DR sequences, CRISPR systems from CoNS also present sequences for the casl gene that differ from on type to the other. A comparison between the cas1 gene sequences from all the strains analyzed in this work showed that when the sequences from both type II and type III cas 1 are aligned together, they present an identity as low as $29 \%$ (data not shown). However, when the sequences are analyzed in their own separate CRISPR-type groups, this value increases to $54 \%$ for type II cas 1 , and to $70 \%$ for type III. These differences lead us to design primers to cas 1 of each group separately, so that the amplification of that gene could be performed in a multiplex PCR reaction that allowed us not only to detect more efficiently strains harboring that CRISPR marker gene, but also permitted their differentiation according to the type of system (Figure 4). In addition, the reaction allows the identification of strains containing more than one type of CRISPR system in its genome, as in the case observed computationally for the S. schleiferi TSCC54 strain, described above.

Because S. haemolyticus is one of the most important CoNS and no CRISPRs have been described to the species so far, the multiplex PCR reaction was employed in 59 clinical strains of this species previously shown by our group to be phenotypic and genetically diverse (Rossi et al., 2016b). The cas1 gene was amplified in only $3 \%$ of the strains (2/59), namely MD03 and MD25. The proportion found is the same observed in the in silico analysis for the species. However, while in the genome available 


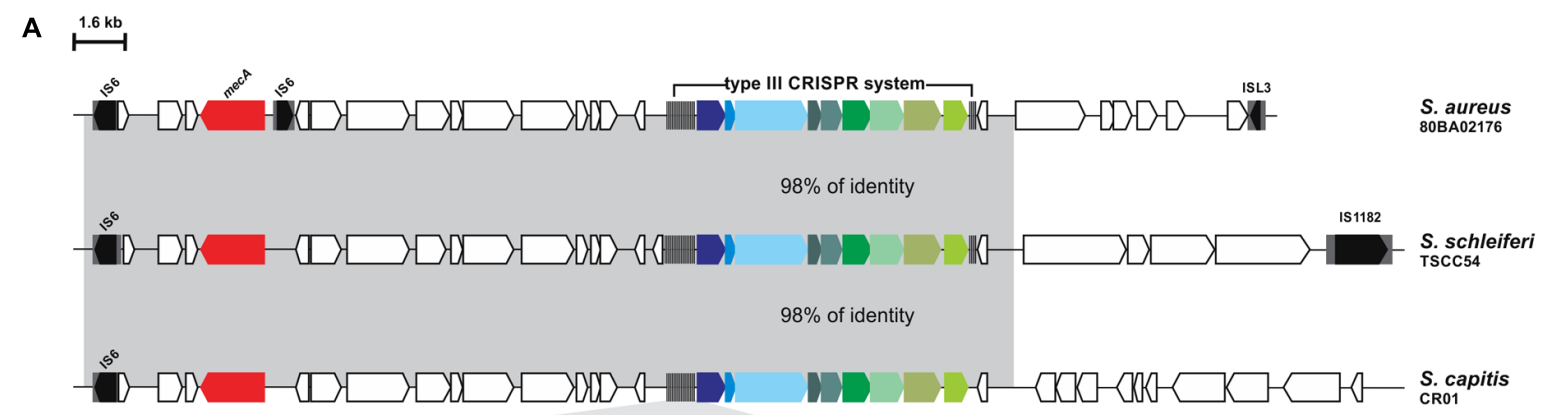

B

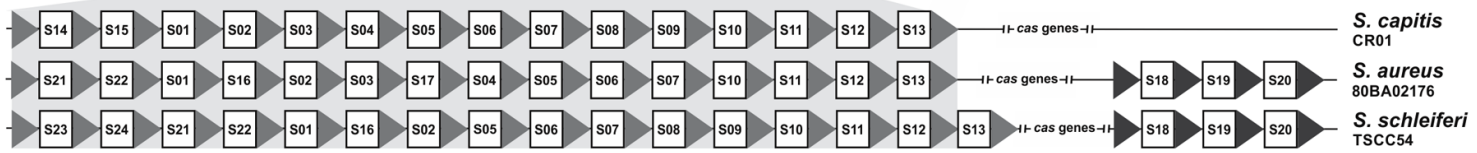

C

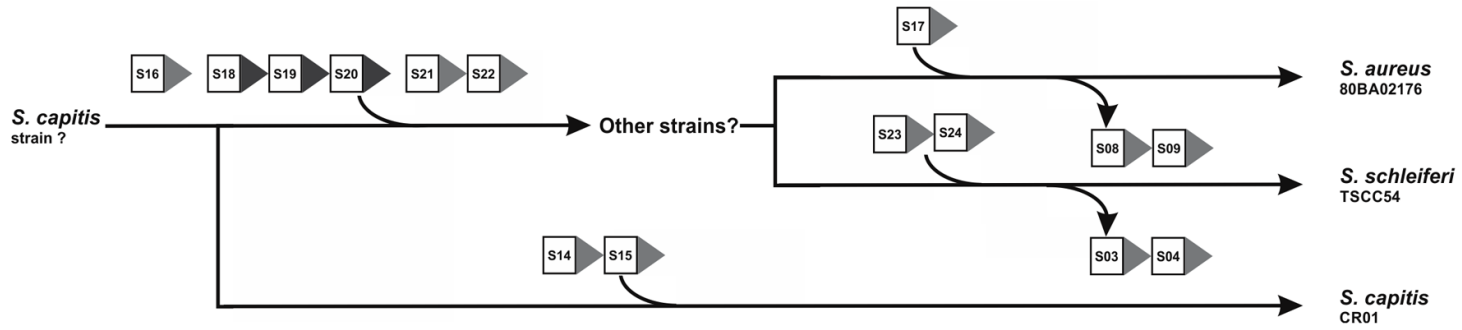

FIGURE 3 | CRISPR systems within SCCmec elements. (A) Comparison of the genetic context of the CRISPR-containing SCCmec of S. schleiferi TSCC54 and S. capitis CR01 analyzed in this work and S. aureus 80BA02176. The mecA gene is highlighted in red. (B) Analysis of the spacer sequences - squares - present in each CRISPR show that many of them are identical. (C) Hypothesis of the history of SCCmec-mediated CRISPR/Cas system transference between S. capitis CR01, S. aureus 80BA02176 and S. schleiferi TSCC54 and the acquisition of new spacers.

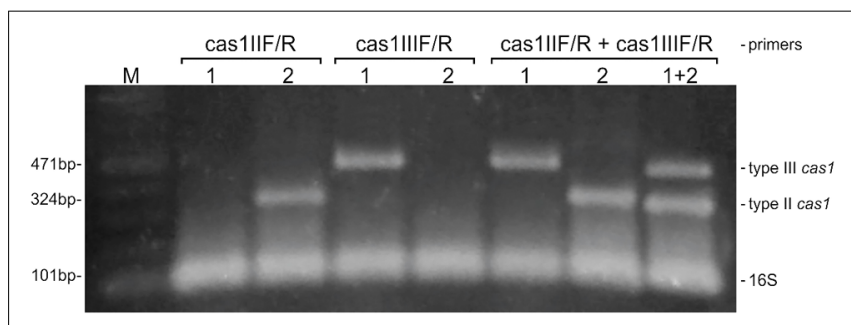

FIGURE 4 | A multiplex PCR for screening and typing CRISPR/Cas systems in coagulase-negative staphylococci. The multiplex was designed to detect the universal cas1 gene, and to differentiate than between those belonging to types II and III CRISPR systems. The detection of the 16S rRNA is employed as an endogenous control of the reaction. S. epidermidis strains RP62A (1) and $10 \mathrm{~L}$ (2) were used as positive controls for the amplification of cas 1 of types II and III, respectively. M: molecular weight marker.

for S. haemolyticus W75 the CRISPR system belongs to the type II, in both of our clinical strains the amplicon size indicates they belong to type III (data not shown).

\section{The cas1 Gene Is Expressed during All Phases of S. haemolyticus Growth}

To get an insight on the functionality of the CRISPR systems found in the screening, we evaluated the expression of the cas 1 gene in strains MD03 and MD05 during exponential growth, stationary phase and in the biofilm form. As expected for a surveillance-related gene, the expression of cas 1 was observed in all situations (Figure 5).

\section{DISCUSSION}

Coagulase-negative staphylococci are advocated to act as resistance and virulence gene reservoirs, but this role could be contradicted by estimates that CRISPR/Cas systems are present in the genomes of more than $40 \%$ of bacteria (Sorek et al., 2008), since these systems greatly interfere with plasmid uptake in staphylococci (Marraffini and Sontheimer, 2008; Cao et al., 2016). However, recent studies indicate that this abundance may be considerably lower in major bacterial lineages that have no cultivated representatives (Burstein et al., 2016). Also consistent with the evidences of constant horizontal gene transfer between staphylococci, a recent study with 636 Staphylococcus aureus strains isolated from hospitals in China found by PCR only 6 (close to $1 \%$ ) strains with a complete arrange of cas genes (Cao et al., 2016).

CRISPR abundance could also be overestimated, as a number of very small "questionable" CRISPRs can be detected computationally, but need to be critically investigated for the presence of cas genes. Here we found that $15 \%$ of the strains 


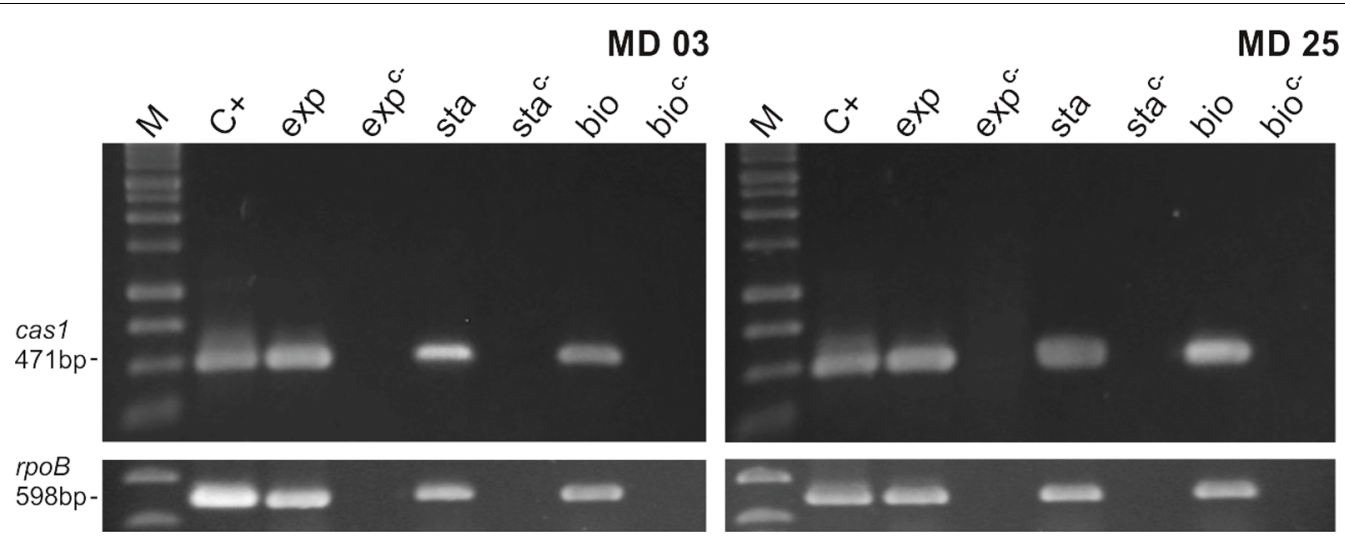

FIGURE 5 | Expression of cas 1 in S. haemolyticus clinical strains MD03 and MD25. The expression of type III cas 1 in the clinical strains that were positive for the multiplex PCR reaction was evaluated during exponential growth (exp), stationary phase (sta) and biofilm formation (bio). M: molecular weight marker, C+: positive control (genomic DNA) and C-: negative control (RNA that was subjected to the cDNA synthesis reaction, but without the addition of the Reverse Transcriptase). The expression of the rpoB gene was used as and endogenous control.

investigated contain real CRISPRs, but we believe that this number could still be overestimated among CoNS, as most of the CRISPR-containing S. schleiferi strains, 1360-13, 2142-05, 2317-03, and 5909-02 were isolated by the same group in the United States (Misic et al., 2015). The largest and more varied group of sequences available are from $S$. epidermidis and S. haemolyticus, and both showed to have a considerably lower proportion of CRISPR systems. These two are the most significant species of CoNS and account substantially for foreign body-related infections and infections in preterm newborns (Becker et al., 2014). They also represent a therapeutic challenge due to the large proportion of methicillin-resistant strains and the great number of antimicrobial resistance genes that they usually harbor (Becker et al., 2014; Czekaj et al., 2015).

Six types of CRISPR systems are virtually accepted (Luo et al., 2016), although most of the bacteria and archaea contain either systems of types I, II, or III, with type II being mostly found in bacteria (Makarova et al., 2015). A metagenomic study has recently revealed the existence of two novel CRISPR-Cas systems containing new Cas proteins, named as CasX and CasY (Cloney, 2017). But like types IV and V systems, their mechanism of action still have to be elucidated. Here we show that CoNS contain either CRISPR-Cas systems of type II (containing Cas1-2 and its signature Cas9 protein), or type III (containing the genes for the proteins Cas1-2, Cas10, Cas6, and Csm2-6). The fact that many of them are surrounded by insertion sequences indicates that they can potentially be transferred horizontally and be eventually lost in the absence of a selective pressure. However, because most of the genomes analyzed are available in the form of draft sequences, deeper analysis of the surroundings of these CRISPRs is not possible.

On the other hand, it is undeniable the existence of CRISPRs within SCCmec elements in two strains studied (S. capitis CR01 and S. schleiferi TSCC54). Even more remarkable is the fact that the sequence of these strains spacers allowed us to observe that these systems have a common origin and that they were transferred between strains and species. This is not only one more evidence of horizontal gene transfer between CoNS and S. aureus, but also between human and animal strains, as $S$. schleiferi TSCC54 was isolated from the skin of a dog in Japan. Even though the species can be recovered from both humans and dogs, it is only clinically significant to the latter, for causing various disease states such as pyoderma, otitis, and urinary tract infections (Sasaki et al., 2015).

Then, the diversity of spacers, as described above, allows tracking the history of genetic material between different strains, species and hosts. Meanwhile, for the bacteria, the more diverse their spacers are, the more efficient is the defense of the population against mobile genetic elements (Lopatina and Sorek, 2016). Most spacers from CoNS are from an unknown origin, which once more reflects the general under-sampling of phage and plasmid sequences and in consequence, their availability in public databases (Mojica et al., 2005). In addition, the fact that some strains contain spacers derived from plasmids found in different species evidences that those CRISPR-containing cells indeed restrict horizontal gene transfer between staphylococci, in accordance to experimental evidences (Marraffini and Sontheimer, 2008; Cao et al., 2016).

Screening for CRISPR-positive strains will help understanding the importance of genetic exchange within that population and, when they are present, it will also help tracking the dynamics of that transfer, by analyzing the sequence of the spacers. Moreover, identifying these systems in species for which no CRISPR elements have been described could increase the repertoire of natural Cas proteins that recognize different PAMs. Choosing the more suitable Cas to perform molecular edition will hopefully facilitate the manipulation of genomes for species to which the systems available are not efficient, especially in prokaryotes (Luo et al., 2016).

The cas 1 gene is ideal for screening purposes for being the only one that is virtually in every CRISPR/Cas system described so far. The cas 2 gene is also widespread, but it is not present in one of the recently described systems (Cloney, 2017). The multiplex PCR developed in here overcomes the sequence polymorphisms 
observed for the cas1 gene among CoNS, being a suitable strategy for identifying and typing CRISPRs among this group of bacteria. Because $S$. haemolyticus is an important reservoir of several antimicrobial resistance genes that can be transferred to S. aureus (Czekaj et al., 2015; Rossi et al., 2016a), we expected that clinical strains of this species would mostly lack CRISPR systems in their genomes. Indeed, our search for the cas 1 gene resulted in a proportion of positive strains as little as that observed by bioinformatics. We also observed that the expression of casl takes place in all growth phases of S. haemolyticus, as it would be expected for a system responsible for surveilling the presence of genetic invaders. However, in some bacteria, like E. coli, envelope stress is a trigger for CRISPR expression (Perez-Rodriguez et al., 2011); and in Myxococcus xanthus the cas genes are located in a larger operon, that is also responds to stressful conditions (Viswanathan et al., 2007). Future studies will elucidate whether biofilm formation, an important virulence determinant of S. haemolyticus, influences the expression of the CRISPR and its associated genes.

\section{CONCLUSION}

This work shows that the abundance of CRISPR/Cas systems in CoNS is considerably lower than the average expected for bacteria in general, which is in accordance with their role as gene reservoirs. However, when present, the sequence of the CRISPR spacers evidence and give an insight on the

\section{REFERENCES}

Amitai, G., and Sorek, R. (2016). CRISPR-Cas adaptation: insights into the mechanism of action. Nat. Rev. Microbiol. 14, 67-76. doi: 10.1038/nrmicro. 2015.14

Asaad, A. M., Ansar Qureshi, M., and Mujeeb Hasan, S. (2016). Clinical significance of coagulase-negative staphylococci isolates from nosocomial bloodstream infections. Infect. Dis. 48, 356-360. doi: 10.3109/23744235.2015.1122833

Barbier, F., Ruppe, E., Hernandez, D., Lebeaux, D., Francois, P., Felix, B., et al. (2010). Methicillin-resistant coagulase-negative staphylococci in the community: high homology of SCCmec IVa between Staphylococcus epidermidis and major clones of methicillin-resistant Staphylococcus aureus. J. Infect. Dis. 202, 270-281. doi: 10.1086/653483

Barrangou, R., and Horvath, P. (2017). A decade of discovery: CRISPR functions and applications. Nat. Microbiol. 2, 17092. doi: 10.1038/nmicrobiol.2017.92

Barros, E. M., Ceotto, H., Bastos, M. C., Dos Santos, K. R., and GiambiagideMarval, M. (2012). Staphylococcus haemolyticus as an important hospital pathogen and carrier of methicillin resistance genes. J. Clin. Microbiol. 50, 166-168. doi: 10.1128/JCM.05563-11

Becker, K., Heilmann, C., and Peters, G. (2014). Coagulase-negative staphylococci. Clin. Microbiol. Rev. 27, 870-926. doi: 10.1128/CMR.00109-13

Burstein, D., Harrington, L. B., Strutt, S. C., Probst, A. J., Anantharaman, K., and Thomas, B. C. (2017). New CRISPR-Cas systems from uncultivated microbes. Nature 542, 237-241. doi: 10.1038/nature21059

Burstein, D., Sun, C. L., Brown, C. T., Sharon, I., Anantharaman, K., and Probst, A. J. (2016). Major bacterial lineages are essentially devoid of CRISPR-Cas viral defence systems. Nat. Commun. 7:10613. doi: 10.1038/ncomms10613

Cao, L., Gao, C. H., Zhu, J., Zhao, L., Wu, Q., Li, M., et al. (2016). Identification and functional study of type III-A CRISPR-Cas systems in clinical isolates of Staphylococcus aureus. Int. J. Med. Microbiol. 306, 686-696. doi: 10.1016/j.ijmm. 2016.08.005 dynamics of horizontal genetic transfer among staphylococci, which undeniably happens between strains, species, and hosts.

\section{AUTHOR CONTRIBUTIONS}

CR conceived the study, designed and performed experiments, purchased materials, and wrote the manuscript. TS-S designed and tested the multiplex PCR reactions. AA-A helped with conceiving of the study and manuscript draft. MG-dM conceived the study, purchased materials, and participated in the study's design and coordination. All authors read and approved the final manuscript.

\section{FUNDING}

This work was supported by Brazilian grants, FAPERJ (grant E-26/203.037/2016), CNPq (grant 304.506/2014-1) and CAPESProex (grant 23038.003823/2014-79). CR is recipient of postdoctoral scholarship from FAPERJ (grants E-26/202.799/2016 and E-26/202.800/2016).

\section{SUPPLEMENTARY MATERIAL}

The Supplementary Material for this article can be found online at: http://journal.frontiersin.org/article/10.3389/fmicb. 2017.01545/full\#supplementary-material

Chen, H., Liu, Y., Zhao, C., Xiao, D., Zhang, J., Zhang, F., et al. (2013). Comparative proteomics-based identification of genes associated with glycopeptide resistance in clinically derived heterogeneous vancomycinintermediate Staphylococcus aureus strains. PLoS ONE 8:e66880. doi: 10.1371/journal.pone.0066880

Cloney, R. (2017). Metagenomics: uncultivated microbes reveal new CRISPR-Cas systems. Nat. Rev. Genet. 18, 146. doi: 10.1038/nrg.2017.1

Crooks, G. E., Hon, G., Chandonia, J. M., and Brenner, S. E. (2004). WebLogo: a sequence logo generator. Genome Res. 14, 1188-1190. doi: 10.1101/gr.849004

Czekaj, T., Ciszewski, M., and Szewczyk, E. M. (2015). Staphylococcus haemolyticus - an emerging threat in the twilight of the antibiotics age. Microbiology 161, 2061-2068. doi: 10.1099/mic.0.000178

Drancourt, M., and Raoult, D. (2002). rpoB gene sequence-based identification of Staphylococcus species. J. Clin. Microbiol. 40, 1333-1338. doi: 10.1128/JCM.40. 4.1333-1338.2002

Flemming, H. C., Wingender, J., Szewzyk, U., Steinberg, P., Rice, S. A., and Kjelleberg, S. (2016). Biofilms: an emergent form of bacterial life. Nat. Rev. Microbiol. 14, 563-575. doi: 10.1038/nrmicro.2016.94

Gill, S. R., Fouts, D. E., Archer, G. L., Mongodin, E. F., Deboy, R. T., and Ravel, J. (2005). Insights on evolution of virulence and resistance from the complete genome analysis of an early methicillin-resistant Staphylococcus aureus strain and a biofilm-producing methicillin-resistant Staphylococcus epidermidis strain. J. Bacteriol. 187, 2426-2438. doi: 10.1128/JB.187.7.2426-2438. 2005

Golding, G. R., Bryden, L., Levett, P. N., McDonald, R. R., Wong, A., and Graham, M. R. (2012). Whole-genome sequence of livestock-associated st398 methicillin-resistant Staphylococcus aureus isolated from humans in Canada. J. Bacteriol. 194, 6627-6628. doi: 10.1128/JB.01680-12

Grissa, I., Vergnaud, G., and Pourcel, C. (2007). CRISPRFinder: a web tool to identify clustered regularly interspaced short palindromic repeats. Nucleic Acids Res. 35, W52-W57. doi: 10.1093/nar/gkm360 
Gruber, A. R., Lorenz, R., Bernhart, S. H., Neubock, R., and Hofacker, I. L. (2008). The Vienna RNA websuite. Nucleic Acids Res. 36, W70-W74. doi: 10.1093/nar/ gkn188

Lopatina, A., and Sorek, R. (2016). CRISPR-Cas: Spacer diversity determines the efficiency of defense. Curr. Biol. 26, R683-R685. doi: 10.1016/j.cub.2016. 05.034

Luo, M. L., Leenay, R. T., and Beisel, C. L. (2016). Current and future prospects for CRISPR-based tools in bacteria. Biotechnol. Bioeng. 113, 930-943. doi: 10.1002/ bit.25851

Makarova, K. S., Wolf, Y. I., Alkhnbashi, O. S., Costa, F., Shah, S. A., and Saunders, S. J. (2015). An updated evolutionary classification of CRISPR-Cas systems. Nat. Rev. Microbiol. 13, 722-736. doi: 10.1038/nrmicro3569

Marraffini, L. A., and Sontheimer, E. J. (2008). CRISPR interference limits horizontal gene transfer in staphylococci by targeting DNA. Science 322, 1843-1845. doi: 10.1126/science.1165771

Misic, A. M., Cain, C. L., Morris, D. O., Rankin, S. C., and Beiting, D. P. (2015). Complete genome sequence and methylome of Staphylococcus schleiferi, an important cause of skin and ear infections in veterinary medicine. Genome Announc. 3, e01011-15. doi: 10.1128/genomeA.01011-15

Mojica, F. J., Diez-Villasenor, C., Garcia-Martinez, J., and Soria, E. (2005). Intervening sequences of regularly spaced prokaryotic repeats derive from foreign genetic elements. J. Mol. Evol. 60, 174-182. doi: 10.1007/s00239-0040046-3

Otto, M. (2013). Coagulase-negative staphylococci as reservoirs of genes facilitating MRSA infection: Staphylococcal commensal species such as Staphylococcus epidermidis are being recognized as important sources of genes promoting MRSA colonization and virulence. Bioessays 35, 4-11. doi: 10.1002/bies. 201200112

Perez-Rodriguez, R., Haitjema, C., Huang, Q., Nam, K. H., Bernardis, S., Ke, A., et al. (2011). Envelope stress is a trigger of CRISPR RNA-mediated DNA silencing in Escherichia coli. Mol. Microbiol. 79, 584-599. doi: 10.1111/j.13652958.2010.07482.x

Ronquist, F., Teslenko, M., van der Mark, P., Ayres, D. L., Darling, A., and Hohna, S. (2012). MrBayes 3.2: efficient Bayesian phylogenetic inference and model choice across a large model space. Syst. Biol. 61, 539-542. doi: 10.1093/ sysbio/sys029

Rossi, C. C., da Silva Dias, I., Muniz, I. M., Lilenbaum, W., and GiambiagideMarval, M. (2017). The oral microbiota of domestic cats harbors a wide variety of Staphylococcus species with zoonotic potential. Vet. Microbiol. 201, 136-140. doi: 10.1016/j.vetmic.2017.01.029

Rossi, C. C., Ferreira, N. C., Coelho, M. L., Schuenck, R. P., Bastos Mdo, C., and Giambiagi-deMarval, M. (2016a). Transfer of mupirocin resistance from Staphylococcus haemolyticus clinical strains to Staphylococcus aureus through conjugative and mobilizable plasmids. FEMS Microbiol. Lett. 363:fnw121. doi: $10.1093 /$ femsle/fnw121
Rossi, C. C., Santos-Gandelman, J. F., Barros, E. M., Alvarez, V. M., Laport, M. S., and Giambiagi-deMarval, M. (2016b). Staphylococcus haemolyticus as a potential producer of biosurfactants with antimicrobial, anti-adhesive and synergistic properties. Lett. Appl. Microbiol. 63, 215-221. doi: 10.1111/lam. 12611

Rutherford, K., Parkhill, J., Crook, J., Horsnell, T., Rice, P., Rajandream, M. A., et al. (2000). Artemis: sequence visualization and annotation. Bioinformatics 16, 944-945.

Sasaki, T., Tsubakishita, S., Kuwahara-Arai, K., Matsuo, M., Lu, Y. J., Tanaka, Y., et al. (2015). Complete genome sequence of methicillin-resistant Staphylococcus schleiferi strain TSCC54 of canine origin. Genome Announc. 3, e01268-15. doi: 10.1128/genomeA.01268-15

Sievers, F., and Higgins, D. G. (2014). Clustal omega. Curr. Protoc. Bioinformatics 48, 1.25.1-1.25.33. doi: 10.1002/0471250953.bi0313s48

Siguier, P., Varani, A., Perochon, J., and Chandler, M. (2012). Exploring bacterial insertion sequences with ISfinder: objectives, uses, and future developments. Methods Mol. Biol. 859, 91-103. doi: 10.1007/978-1-61779-603-6_5

Sorek, R., Kunin, V., and Hugenholtz, P. (2008). CRISPR-a widespread system that provides acquired resistance against phages in bacteria and archaea. Nat. Rev. Microbiol. 6, 181-186. doi: 10.1038/nrmicro1793

van der Oost, J., Westra, E. R., Jackson, R. N., and Wiedenheft, B. (2014). Unravelling the structural and mechanistic basis of CRISPR-Cas systems. Nat. Rev. Microbiol. 12, 479-492. doi: 10.1038/nrmicro3279

Viswanathan, P., Murphy, K., Julien, B., Garza, A. G., and Kroos, L. (2007). Regulation of dev, an operon that includes genes essential for Myxococcus xanthus development and CRISPR-associated genes and repeats. J. Bacteriol. 189, 3738-3750. doi: 10.1128/JB.00187-07

Vuong, C., and Otto, M. (2002). Staphylococcus epidermidis infections. Microbes Infect. 4, 481-489. doi: 10.1016/S1286-4579(02)01563-0

Conflict of Interest Statement: The authors declare that the research was conducted in the absence of any commercial or financial relationships that could be construed as a potential conflict of interest.

The reviewer PM-A and handling Editor declared their shared affiliation, and the handling Editor states that the process nevertheless met the standards of a fair and objective review.

Copyright (C) 2017 Rossi, Souza-Silva, Araújo-Alves and Giambiagi-deMarval. This is an open-access article distributed under the terms of the Creative Commons Attribution License (CC BY). The use, distribution or reproduction in other forums is permitted, provided the original author(s) or licensor are credited and that the original publication in this journal is cited, in accordance with accepted academic practice. No use, distribution or reproduction is permitted which does not comply with these terms. 